\title{
Assessing leaf scale measurement for nitrogen content of oil palm: performance of discriminant analysis and support vector machine classifiers
}

\begin{abstract}
Nitrogen $(\mathrm{N})$ is a crucial element in sustaining oil palm production. However, assessing $\mathrm{N}$ status of tall perennial crops such as oil palm is complex and not as straightforward as assessing annual crops, due to complex $\mathrm{N}$ partitioning, age, and larger amounts of respiratory loads. Hence, the objectives of this study were to evaluate the potential of spectral measurements obtained from leaf scale and machine learning approaches as a rapid tool for quantifying oil palm $\mathrm{N}$ status. This study involved assessing the performance of discriminant analysis (DA) and Support Vector Machine (SVM) classifiers for discriminating spectral bands sensitive to $\mathrm{N}$ sufficiency levels and comparing the predictive accuracy of DA and SVM for classifying $\mathrm{N}$ status of immature and mature oil palms. The experiment was conducted on immature Tenera seedlings (13 months old) and mature Tenera palm stands (9 and 12 years old) that were arranged in Randomized Complete Block Design with treatments varied from 0 to $2 \mathrm{~kg} \mathrm{~N}$. Generally, the discriminant function of both classifiers was agedependent. A clear trade-off between the classifiersô number of spectral bands and their accuracies was observed; the DA with a larger number of optimal spectral bands could discriminate $\mathrm{N}$ sufficiency levels of all maturity classes with higher accuracies compared to the SVM, yet the latter could produce reasonable accuracies with a lesser number of spectral bands. N status of all maturity classes could be classified satisfactorily with SVM (71ї 88\%) via the satellite-simulated blue and green bands, signifying the possibility to develop spectral index or an $\mathrm{N}$-sensitive sensor for oil palm.
\end{abstract}

Keyword: Nitrogen content; Oil palm; Support vector machine; Discriminant analysis; Leaf scale 